\title{
Visualization positioning-guided biopsy of suspicious breast microcalcifications: a retrospective cohort study
}

\author{
Liulu Zhang $^{1 \#}$, Minyi Cheng ${ }^{1 \#}$, Yuanqi Chen ${ }^{1,2 \#}$, Xiaosheng Zhuang ${ }^{1}$, Ciqiu Yang ${ }^{1}$, Fei Ji ${ }^{1}$, Hongfei Gao ${ }^{1}$, \\ Mei Yang', Teng Zhu' ${ }^{1}$, Jieqing Li ${ }^{1}$, Kun Wang ${ }^{1}$ \\ ${ }^{1}$ Department of Breast Cancer, Cancer Center, Guangdong Provincial People's Hospital, Guangdong Academy of Medical Sciences, Guangzhou, \\ China; ${ }^{2}$ The Second School of Clinical Medicine, Southern Medical University, Guangzhou, China \\ Contributions: (I) Conception and design: L Zhang, M Cheng, Y Chen; (II) Administrative support: K Wang; (III) Provision of study materials or \\ patients: C Yang, F Ji, H Gao, M Yang, T Zhu, J Li; (IV) Collection and assembly of data: X Zhuang; (V) Data analysis and interpretation: L Zhang; (VI) \\ Manuscript writing: All authors; (VII) Final approval of manuscript: All authors. \\ \#These authors contributed equally to this work. \\ Correspondence to: Kun Wang. Guangdong Provincial People's Hospital, Guangdong Academy of Medical Sciences, Guangzhou 510080, China. \\ Email: wangkun@gdph.org.cn.
}

\begin{abstract}
Background: At present, most histological evaluations of microcalcifications without a mass are performed using X-ray guided hook wire localization or vacuum-assisted stereotactic biopsy (VASB), but there are still several limitations to these techniques. Therefore, we designed a visualization positioning technique based on three directions of mammography to accurately locate suspected microcalcifications to guide the biopsy.

Methods: We retrospectively analyzed consecutive patients with suspicious microcalcifications who underwent visualization positioning-guided biopsy (VPB) from June 1, 2016, to June 1, 2021. The visualization positioning technique was performed using an electronic ruler to measure the vertical distance from the microcalcification core to the vertical lines on mammography.

Results: A total of 133 patients (median age 46 years; range, 22-87 years) who underwent VPB were included in our study. Among the 133 cases of microcalcifications based on pathological results, 104 were benign, 14 were high risk, and 15 were malignant. In 124 (93.2\%) patients, microcalcification was confirmed during the first round of VPB specimen analysis. Only 6 (4.5\%) and 3 (2.3\%) patients underwent second and third extended resections, respectively, as the resected specimens did not contain microcalcifications. Four patients $(3.0 \%)$ with malignant biopsy results underwent a subsequent operation. Two patients with DCIS underwent mastectomy and sentinel lymph node biopsy because of diffuse calcification. One patient had no residual cancer, and the other was upgraded to invasive ductal carcinoma (IDC). Two patients with IDC underwent breast-conserving surgery and mastectomy with sentinel lymph node biopsy.

Conclusions: VPB can be used to evaluate breast microcalcifications when a mass is not present, making it an effective diagnostic technique.
\end{abstract}

Keywords: Breast; microcalcification; biopsy

Submitted Jul 09, 2021. Accepted for publication Oct 14, 2021.

doi: $10.21037 / \mathrm{atm}-21-4496$

View this article at: https://dx.doi.org/10.21037/atm-21-4496

\section{Introduction}

Ultrasound and mammography are usually recommended for breast tumor screening. Surgery-based comprehensive treatment (including chemotherapy, radiotherapy, endocrine therapy and targeted therapy) is the main treatment strategy for breast cancer. Patients with early-stage tumors have a better prognosis than those with late-stage tumors.

Polymorphic or aggregated microcalcifications may be early indications of breast cancer $(1,2)$. These lesions 
are usually classified into more than 4 categories by the Breast Imaging Reporting and Data System (BI-RADS) and need to be confirmed histologically (3). Mammography is a very sensitive imaging method for detecting microcalcifications. However, microcalcifications without a mass are usually not recognized by clinical palpation or ultrasound (4). Therefore, conventional surgery or ultrasound-guided biopsy cannot be used to accurately evaluate microcalcifications.

At present, most histological evaluations of microcalcifications are performed using $\mathrm{X}$-ray guided hook wire localization or vacuum-assisted stereotactic biopsy (VASB). However, stereotactic devices are expensive and require an experienced radiologist. In addition, patients will be exposed to radiation more than once during the operation. Moreover, VASB is not suitable for patients with small breasts or lesions located at the edge of the breast, as well as those who have undergone breast augmentation surgery. Previous studies showed that the underestimation rate of malignant tumors was between $0 \%$ and 29\% when the diagnosis of VASB was flat epithelial atypia (FEA) or atypical ductal hyperplasia (ADH) (5-12). For patients with VASB-diagnosed high-risk or malignant lesions or with residual microcalcification, surgical resection is still needed.

For nonpalpable breast lesions, hook wire-guided localization is also a common approach $(13,14)$. The microcalcifications shown only on mammography can be located only under mammographic guidance. Nevertheless, the patient is exposed to radiation several times during localization, and the position of the guide wire may change during patient activity.

Therefore, we designed a visualization positioning technique based on three directions of mammography to accurately locate suspected microcalcifications on the skin and guide the biopsy. Our study provides a new alternative biopsy method for clinical practice. We present the following article in accordance with the STROBE reporting checklist (available at https://dx.doi. org/10.21037/atm-21-4496).

\section{Methods}

\section{Patients}

This retrospective cohort study included consecutive patients who received visualization positioning-guided biopsy (VPB) in the Department of Breast Cancer, Guangdong Provincial People's Hospital between June 1,
2016, and June 1, 2021 due to suspicious microcalcifications detected by mammography. Patients with incomplete imaging or pathological data were excluded from the analyses. Before biopsy, microcalcifications were confirmed by mammography, and breast palpation and ultrasound were performed. All microcalcifications associated with the mass were excluded. Two experienced breast radiologists performed a retrospective evaluation of each suspected microcalcification on the high-resolution digital mammography screen, and lesions were classified and morphologically described according to the 5 th edition of the BI-RADS (3). In our study, biopsies were also performed in some patients with BI-RADS 0 or 3 lesions due to a family history of breast cancer or a strong desire of the patients. All procedures involving human participants were performed in accordance with the Declaration of Helsinki (as revised in 2013). This study was approved by the Research Ethics Committee of Guangdong Provincial People's Hospital, Guangdong Academy of Medical Sciences (No. GDREC 2018253H). All patients signed a written informed consent form before the operation after fully understanding the possible risks and complications.

\section{VPB procedure}

Before the operation, digital mammography (uMammo $790 \mathrm{i}$, UNITED IMAGING) was performed in the cranio-caudal (CC), lateral-medio (LM), and mediolateral oblique (MLO) views. The surgeon confirmed the locations of the microcalcifications in the CC, LM, and MLO views. On mammography, we measured the distance from the microcalcification core to the vertical line through the papilla (CC') using an electronic ruler and examined whether the microcalcification was located in the inner or outer portion. Similarly, the distance from the microcalcification core to the vertical line through the nipple (LM' and MLO') was measured in the LM and MLO views of the mammogram (Figure $1 A-1 D$ ). The patients were in the standing position and raised both hands in the same position while undergoing mammography, and the surgeon made 3 lines parallel to CC', LM', and MLO' according to the measured distance. The location where the 3 lines intersected was defined as the microcalcification site (Figure 1E).

During biopsy, the localized microcalcification area was excised, and the direction of the specimen was marked with a radiopaque metal clip (Figure 2). If specimen mammography showed enough microcalcifications, the 


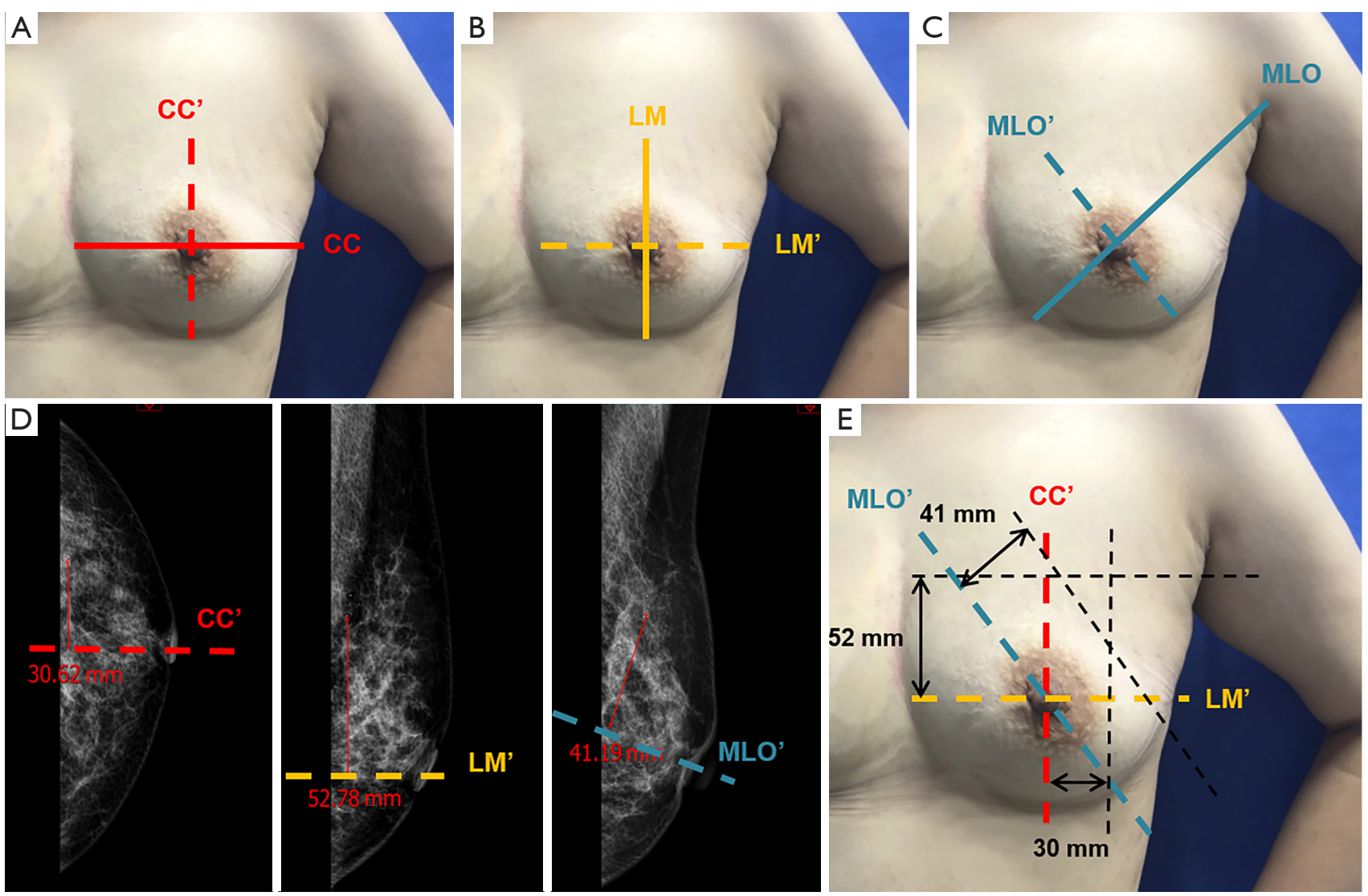

Figure 1 Visualization positioning-guided biopsy for the identification of the location of breast microcalcifications. (A-C) The vertical lines CC', LM', and MLO' were through the nipple in the cranio-caudal (CC), lateral-medio (LM), and medio-lateral oblique (MLO) views. (D) Microcalcifications were detected $30 \mathrm{~mm}$ from the midline in the outer part of the left breast in the CC view, $52 \mathrm{~mm}$ from the midline in the upper part in the LM view, and $41 \mathrm{~mm}$ from the midline in the upper outer part in the MLO view. (E) Microcalcifications were located at the intersection of the 3 lines.
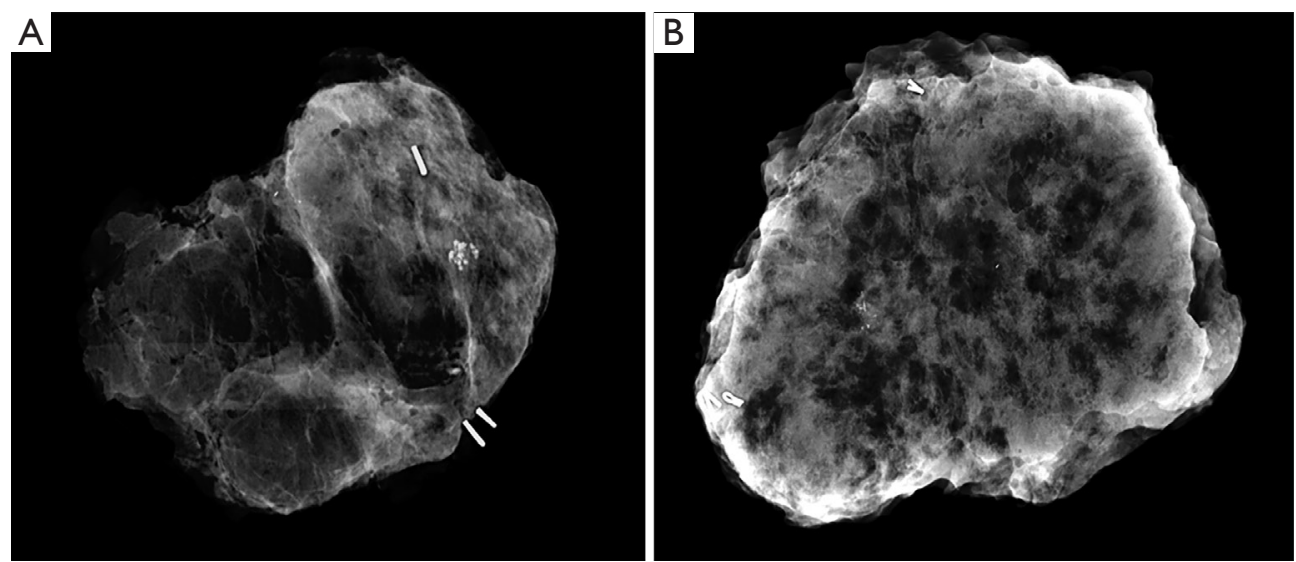

Figure 2 The microcalcification area was excised, and the direction of the specimen was marked with a radiopaque metal clip. Specimen mammography showed that the microcalcification area was included. (A,B) show the microcalcification specimens of two patients. 


\section{Page 4 of 9}

Table 1 Patients' clinicopathological findings and mammographic features $(\mathrm{N}=133)$

\begin{tabular}{lc}
\hline Variable & $\mathrm{n}(\%)$ \\
\hline Age (years), median [range] & $46[28-87]$ \\
Lesion location & $59(44.4)$ \\
Right breast & $74(55.6)$ \\
Left breast & \\
Morphology of microcalcifications & $23(17.3)$ \\
Fine linear/branching & $43(32.3)$ \\
Amorphous & $5(3.8)$ \\
Coarse heterogeneous & $62(46.6)$ \\
Fine pleomorphic & \\
BI-RADS category & $6(4.5)$ \\
0 & $29(21.8)$ \\
3 & $90(67.7)$ \\
4 & $8(6.0)$ \\
5 &
\end{tabular}

BI-RADS, Breast Imaging Reporting and Data System.

operation was completed. However, if microcalcifications were not visible on mammography, the residual cavity of the breast was further expanded until the main calcification was included in the resected specimen.

Tissue samples containing microcalcifications were placed in formalin and processed in a pathology laboratory.

\section{Histopathological evaluation}

Histopathological analysis was performed retrospectively by two professional breast pathologists. The histopathological diagnosis was divided into benign, high risk, and malignant. High-risk lesions included atypical lobular hyperplasia (ALH), ADH, FEA, and lobular intraepithelial neoplasia (LIN). Malignant lesions included ductal carcinoma in situ (DCIS) and invasive carcinoma.

The incisal margins of high-risk and malignant lesions were also detected.

\section{Management after biopsy}

After VPB, the patients were treated according to the results of the histopathological examination. In our protocol, follow-up was recommended for any category

\section{Zhang et al. Visualization positioning for microcalcification biopsy}

of benign, high-risk, and DCIS patients with negative margins. Postoperative reviews were necessary after 3 months and then every 6 months to monitor complications and recurrence.

All patients with a VPB pathology of invasive cancer or with a positive margin needed further surgical operation, and the subsequent operation rate was evaluated. Patients with breast cancer underwent mastectomy or breastconserving surgery combined with sentinel lymph node biopsy or axillary lymph node dissection.

\section{Statistical analysis}

The clinical and pathological data of the included patients were collected from an electronic database and analyzed statistically. Descriptive statistics and univariate analysis were performed. Data are expressed as the frequency and percentage. Statistical Package for Social Sciences (version 20.0) software (SPSS Inc., Chicago, IL, USA) was used for data analysis.

\section{Results}

\section{Patients' clinical and mammographic characteristics}

Of the 151 patients, 18 were excluded due to a lack of imaging or pathological data. A total of 133 patients (median age 46 years; range, 22-87 years) who underwent VPB were included in our study. There were 59 cases $(44.4 \%)$ on the right side and 74 cases $(55.6 \%)$ on the left side. The morphologies of the microcalcifications on mammography were fine linear/branching in 23 patients $(17.3 \%)$, amorphous in $43(32.3 \%)$, coarse heterogeneous in $5(3.8 \%)$, and fine pleomorphic in $62(46.6 \%)$. A total of $29(21.8 \%)$ lesions were classified as BI-RADS category 3, $90(67.7 \%)$ as BI-RADS category 4 , and $8(6.0 \%)$ as BI-RADS category 5, while 6 cases $(4.5 \%)$ of BI-RADS category 0 also underwent biopsy. The patients' clinicopathological findings and mammography features are shown in Table 1.

\section{Histopathological diagnoses}

Among the 133 cases of microcalcifications based on pathological results, 104 were benign, 14 were high risk, and 15 were malignant (Table 2). These 104 cases of benign lesions included 6 histological diagnoses: fibroadenoma (13 cases; 9.8\%), usual ductal hyperplasia (29 cases; $21.8 \%$ ), 
benign stromal calcifications (30 cases; $22.6 \%$ ), intraductal papilloma (12 cases; $9.0 \%)$, columnar cell changes (14 cases; $10.5 \%)$, and mucocele (6 cases; $4.5 \%)$. Among the 14 patients with high-risk lesions, 6 (4.5\%) had ADH, $3(2.3 \%)$ had FEA, and 5 (3.8\%) had LIN. Among the 15 cases of malignant lesions, $13(9.8 \%)$ were DCIS and 2 $(1.5 \%)$ were invasive ductal carcinoma (IDC). According to the final pathological results, the malignancy rates of lesions classified as BI-RADS categories 3, 4, and 5 were $6.9 \%(2 / 29), 12.2 \%$ (11/90), and $25.0 \%$ (2/8), respectively (Table 3).

Table 2 Histopathological diagnoses of microcalcifications $(\mathrm{N}=133)$

\begin{tabular}{lc}
\hline Category & $\mathrm{n}(\%)$ \\
\hline Benign (N=104) & $13(9.8)$ \\
Fibroadenoma & $29(21.8)$ \\
Usual ductal hyperplasia & $30(22.6)$ \\
Benign stromal calcifications & $12(9.0)$ \\
Intraductal papilloma & $14(10.5)$ \\
Columnar cell changes & $6(4.5)$ \\
Mucocele & \\
High risk (N=14) & $6(4.5)$ \\
Atypical ductal hyperplasia (ADH) & $3(2.3)$ \\
Flat epithelial atypia (FEA) & $5(3.8)$ \\
Lobular intraepithelial neoplasia (LIN) & \\
Malignant (N=15) & $13(9.8)$ \\
Ductal carcinoma in situ (DCIS) & $2(1.5)$ \\
Invasive ductal carcinoma (IDC) & \\
\hline
\end{tabular}

\section{$V P B$ and subsequent operations}

The average long and short diameters of the excised microcalcification specimens were $51 \mathrm{~mm}$ (range, $16-90 \mathrm{~mm}$ ) and $39 \mathrm{~mm}$ (range, $12-75 \mathrm{~mm}$ ), respectively. In $124(93.2 \%)$ patients, microcalcifications were confirmed during the first round of VPB analysis (Figure 2). Only 6 $(4.5 \%)$ and $3(2.3 \%)$ patients underwent second and third extended resections, respectively, as the resected specimens did not contain microcalcifications (Table 4).

Four patients $(3.0 \%)$ with malignant biopsy results underwent a subsequent operation. Two patients with DCIS underwent mastectomy and sentinel lymph node biopsy because of diffuse calcification. One patient had no residual cancer, and the other was upgraded to IDC. Two patients with IDC underwent breast-conserving surgery and mastectomy with sentinel lymph node biopsy (Figure 3).

\section{Discussion}

As a tool for breast cancer screening, the widespread use of mammography has increased the detection of suspicious nonpalpable breast lesions (15). Microcalcification is one of the most common and sometimes the only mammographic manifestation of breast cancer. Therefore, it is necessary to evaluate microcalcifications found on mammography. Previous data have shown that approximately $50 \%$ of nonpalpable breast cancers manifest as microcalcifications on mammography. In addition, $80-90 \%$ of DCIS lesions have microcalcifications without a mass $(16,17)$.

However, the properties of microcalcifications cannot be accurately estimated by clinical features and imaging findings. Therefore, a selective histological diagnosis is needed to eliminate the risk of misdiagnosis. It should be

Table 3 Histopathological results of specimens according to the BI-RADS classification

\begin{tabular}{lccccc}
\hline \multirow{2}{*}{ BI-RADS category } & \multicolumn{5}{c}{ Histopathological results } \\
\cline { 2 - 6 } & Benign & High risk & DCIS & Invasive carcinoma & Total \\
\hline 0 & 6 & 0 & 0 & 0 & 6 \\
3 & 27 & 0 & 2 & 0 & 29 \\
4 & 67 & 12 & 11 & 0 & 90 \\
5 & 4 & 2 & 0 & 2 & 8 \\
Total & 104 & 14 & 13 & 2 & 133 \\
\hline
\end{tabular}

BI-RADS, Breast Imaging Reporting and Data System; DCIS, ductal carcinoma in situ. 


\section{Page 6 of 9}

Table 4 Visualization positioning technique-guided biopsy (VPB) and subsequent operations

\begin{tabular}{lc}
\hline Variables & $\mathrm{n}(\%)$ \\
\hline Resected tissue diameter (mm), mean [range] & $51[16-90]$ \\
Long & $39[12-75]$ \\
Short & \\
Additional extended biopsy (times) & $124(93.2)$ \\
0 & $6(4.5)$ \\
1 & $3(2.3)$ \\
2 & \\
Subsequent operation & $129(97.0)$ \\
No & $4(3.0)$ \\
Yes & \\
\hline
\end{tabular}

\section{Zhang et al. Visualization positioning for microcalcification biopsy}

noted that in our study, the malignancy rates of lesions of BI-RADS categories 3, 4, and 5 were $6.9 \%(2 / 29), 12.2 \%$ (11/90), and $25.0 \%(2 / 8)$, respectively. In other words, microcalcifications of BI-RADS category 3 also have a probability of malignancy.

For nonpalpable lesions, hook wire-guided localization biopsy is one option for histopathological evaluation (18). Microcalcifications shown only on mammography can be located only under mammographic guidance. The technique uses a hook wire to locate microcalcifications under X-ray guidance so that the breast gland near the hook wire can be excised during surgery. There are still several limitations to hook wire-guided localization under mammographic guidance. First, the patient is exposed to radiation several times during localization. Moreover, hook wire-guided localization is usually performed by radiologists

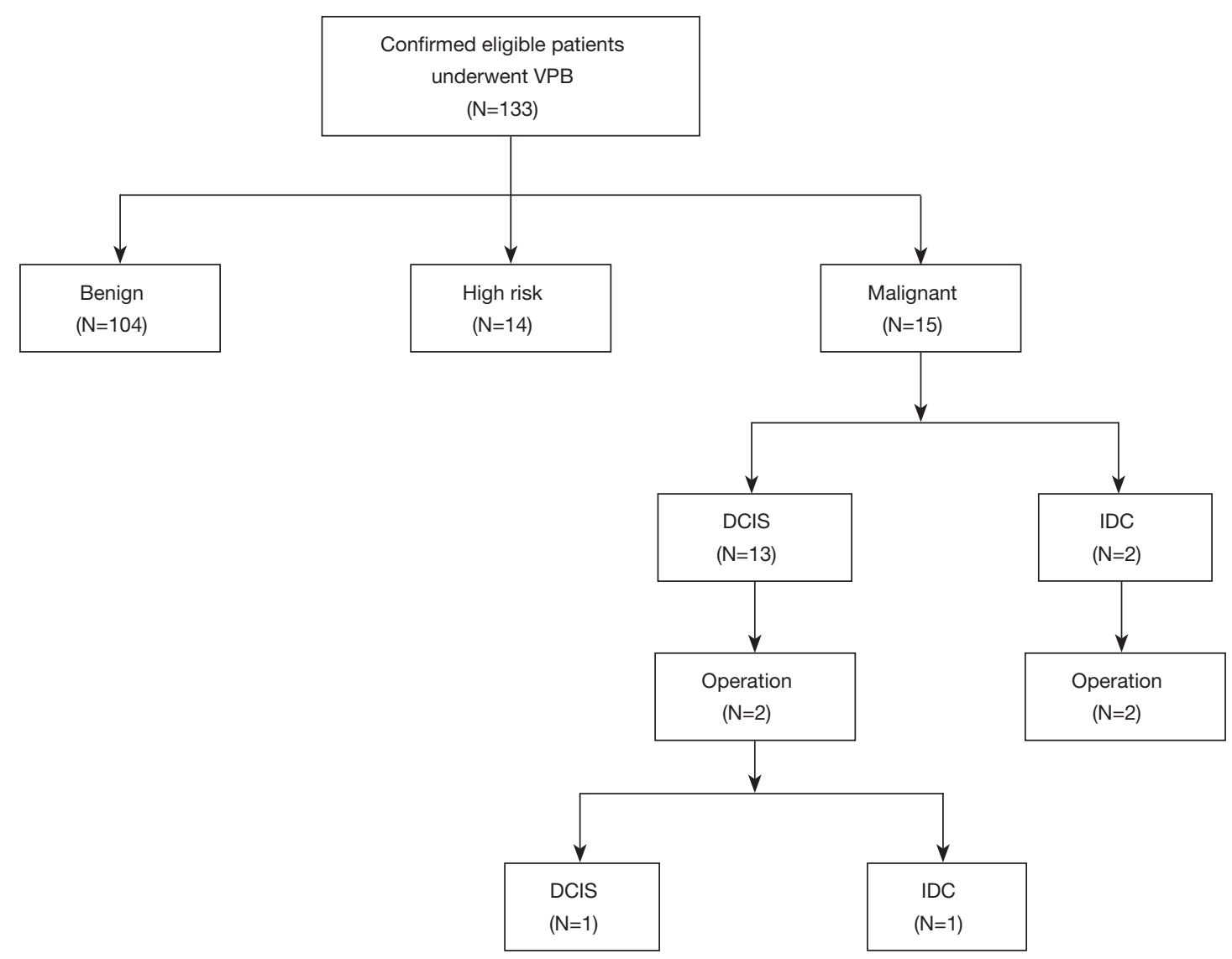

Figure 3 Flowchart of study participants. VPB, Visualization positioning-guided biopsy; DCIS, ductal carcinoma in situ; IDC, Invasive ductal carcinoma. 
before surgery. Therefore, the position of the guide wire may change due to patient movement.

VASB is increasingly being used for the histological diagnosis of suspected microcalcifications. As a routine microcalcification operation, VASB can preclude the need for surgical resection in some patients. VASB is usually performed with the patient in the prone position on a special operating table. It is a safe and accurate tissue sampling method under the guidance of mammography. After the target lesion was determined, $15^{\circ}$ and $215^{\circ}$ stereotactic images were obtained, and the lesion coordinates were calculated. Then, samples were collected through vacuum-assisted blast biopsy, and $\mathrm{X}$-rays were obtained to confirm the existence of calcification. However, the main limitations of VASB are underestimation and the reoperation rate. Histopathological underestimation is defined as a high-risk lesion identified by VASB that is upgraded to DCIS or invasive carcinoma or DCIS that is upgraded to invasive carcinoma after surgical resection (19). In addition, for patients with high-risk or malignant VASB pathology, subsequent surgery is inevitable. At present, there is no consensus on the further treatment of patients with VASB-diagnosed ADH, FEA, or LIN (20). Usually, these patients may need extended resection to remove residual atypical lesions. For patients with malignant tumors after VASB, further breast-conserving surgery or mastectomy and axillary staging are inevitable. Previous studies have reported a reoperation rate ranging from $8.5 \%$ to $62 \%(19,21-23)$.

Microcalcifications were located and measured in the CC, LM, and MLO views on mammography. In the CC view, the location of the lesion was determined to be outer or inner according to the vertical line passing through the nipple. In the LM view, the position was determined to be upper or lower according to the vertical line passing through the nipple. Similarly, in the MLO view, the position was determined to be upper outer or lower inner according to the vertical line of the nipple. Microcalcifications were located at the intersection of the three lines. In this way, the location of microcalcifications on the skin can be easily and accurately marked by electronic measurements.

VPB can locate microcalcifications that are invisible to the naked eye and can assist in biopsy by providing visual skin surface markers. Compared with hook wireguided localization biopsy or VASB, VPB avoids repeat radiation exposure, as only the excised specimens need to be confirmed by X-ray. Moreover, VPB can be completed by surgeons without special equipment and radiologists.
In total, $93.2 \%(124 / 133)$ of patients had calcified tissue samples obtained through a single biopsy. Through singleround VPB combined with margin detection, the surgical treatment of benign, high-risk, and DCIS lesions can be completed at the same time. Only 4 patients $(3.0 \%)$ underwent subsequent operations.

There are several limitations to this technique. Surgeons should have a good understanding of mammography, and it is necessary to practice the identification, measurement, and location of lesions. Of course, VPB is not superior to traditional VASB for identifying microcalcifications. However, our technique will be helpful for cases when VASB is not possible. VPB has a significant advantage over VASB in patients who cannot maintain a prone position for a long period of time, are at risk of bleeding, or have a scattered microcalcification range.

In conclusion, VPB can be used to evaluate breast lesions with microcalcification only, making it an effective diagnostic technique.

\section{Acknowledgments}

Funding: This work was supported by the Medical Scientific Research Foundation of Guangdong Province, China (B2021406).

\section{Footnote}

Reporting Checklist: The authors have completed the STROBE reporting checklist. Available at https://dx.doi. org/10.21037/atm-21-4496

Data Sharing Statement: Available at https://dx.doi. org/10.21037/atm-21-4496

Conflicts of Interest: All authors have completed the ICMJE uniform disclosure form (available at https://dx.doi. org/10.21037/atm-21-4496). The authors have no conflicts of interest to declare.

Ethical Statement: The authors are accountable for all aspects of the work in ensuring that questions related to the accuracy or integrity of any part of the work are appropriately investigated and resolved. All procedures involving human participants were performed in accordance with the Declaration of Helsinki (as revised in 2013). This study was approved by the Research Ethics Committee of Guangdong Provincial People's Hospital, Guangdong 


\section{Page 8 of 9}

Academy of Medical Sciences (No. GDREC 2018253H). All patients signed a written informed consent form before the operation after fully understanding the possible risks and complications.

Open Access Statement: This is an Open Access article distributed in accordance with the Creative Commons Attribution-NonCommercial-NoDerivs 4.0 International License (CC BY-NC-ND 4.0), which permits the noncommercial replication and distribution of the article with the strict proviso that no changes or edits are made and the original work is properly cited (including links to both the formal publication through the relevant DOI and the license). See: https://creativecommons.org/licenses/by-nc-nd/4.0/.

\section{References}

1. Atasoy MM, Tasali N, Çubuk R, et al. Vacuumassisted stereotactic biopsy for isolated BI-RADS 4 microcalcifications: evaluation with histopathology and midterm follow-up results. Diagn Interv Radiol 2015;21:22-7.

2. Gümüş H, Mills P, Fish D, et al. Breast microcalcification: diagnostic value of calcified and non-calcified cores on specimen radiographs. Breast J 2013;19:156-61.

3. American College of Radiology. Breast imaging reporting and data system, 5th edn. Reston, VA: American College of Radiology, 2013.

4. Moon WK, Im JG, Koh YH, et al. US of mammographically detected clustered microcalcifications. Radiology 2000;217:849-54.

5. Kunju LP, Kleer CG. Significance of flat epithelial atypia on mammotome core needle biopsy: Should it be excised? Hum Pathol 2007;38:35-41.

6. Chivukula M, Bhargava R, Tseng G, et al. Clinicopathologic implications of "flat epithelial atypia" in core needle biopsy specimens of the breast. Am J Clin Pathol 2009;131:802-8.

7. Piubello Q, Parisi A, Eccher A, et al. Flat epithelial atypia on core needle biopsy: which is the right management? Am J Surg Pathol 2009;33:1078-84.

8. Senetta R, Campanino PP, Mariscotti G, et al. Columnar cell lesions associated with breast calcifications on vacuum-assisted core biopsies: clinical, radiographic, and histological correlations. Mod Pathol 2009;22:762-9.

9. Ingegnoli A, d'Aloia C, Frattaruolo A, et al. Flat epithelial atypia and atypical ductal hyperplasia: carcinoma underestimation rate. Breast J 2010;16:55-9.
Zhang et al. Visualization positioning for microcalcification biopsy

10. Liberman L, Smolkin JH, Dershaw DD, et al. Calcification retrieval at stereotactic, 11-gauge, directional, vacuumassisted breast biopsy. Radiology 1998;208:251-60.

11. Penco S, Rizzo S, Bozzini AC, et al. Stereotactic vacuumassisted breast biopsy is not a therapeutic procedure even when all mammographically found calcifications are removed: analysis of 4,086 procedures. AJR Am J Roentgenol 2010;195:1255-60.

12. Eby PR, Ochsner JE, DeMartini WB, et al. Frequency and upgrade rates of atypical ductal hyperplasia diagnosed at stereotactic vacuum-assisted breast biopsy: 9-versus 11-gauge. AJR Am J Roentgenol 2009;192:229-34.

13. van Esser S, Hobbelink MG, Peeters PH, et al. The efficacy of 'radio guided occult lesion localization' (ROLL) versus 'wire-guided localization' (WGL) in breast conserving surgery for non-palpable breast cancer: a randomized clinical trial - ROLL study. BMC Surg 2008;8:9.

14. Ahmed M, Douek M. Intra-operative ultrasound versus wire-guided localization in the surgical management of non-palpable breast cancers: systematic review and metaanalysis. Breast Cancer Res Treat 2013;140:435-46.

15. Mei H, Xu J, Yao G, et al. The diagnostic value of MRI for architectural distortion categorized as BI-RADS category $3-4$ by mammography. Gland Surg 2020;9: 1008-18.

16. Bray F, Ferlay J, Soerjomataram I, et al. Global cancer statistics 2018: GLOBOCAN estimates of incidence and mortality worldwide for 36 cancers in 185 countries. CA Cancer J Clin 2018;68:394-424.

17. Ferranti C, Coopmans de Yoldi G, Biganzoli E, et al. Relationships between age, mammographic features and pathological tumour characteristics in non-palpable breast cancer. Br J Radiol 2000;73:698-705.

18. Meyer JE, Smith DN, Lester SC, et al. Large-core needle biopsy of nonpalpable breast lesions. JAMA 1999;281:1638-41.

19. Safioleas PM, Koulocheri D, Michalopoulos N, et al. The value of stereotactic vacuum assisted breast biopsy in the investigation of microcalcifications. A six-year experience with 853 patients. J BUON 2017;22:340-6.

20. Schiaffino S, Massone E, Gristina L, et al. Vacuum assisted breast biopsy (VAB) excision of subcentimeter microcalcifications as an alternative to open biopsy for atypical ductal hyperplasia. Br J Radiol 2018;91:20180003.

21. Liberman L, Gougoutas CA, Zakowski MF, et al. Calcifications highly suggestive of malignancy: comparison of breast biopsy methods. AJR Am J Roentgenol 
2001;177:165-72.

22. Li S, Qu F, Yang Y, et al. Value of stereotactic 11-gauge vacuum-assisted breast biopsy in non-palpable suspicious calcifications: an eight-year single institution experience with 587 patients. Gland Surg 2020;9:1258-66.

23. Yu CC, Cheung YC, Ueng SH, et al. Impact of NonCalcified Specimen Pathology on the Underestimation

Cite this article as: Zhang L, Cheng M, Chen Y, Zhuang X, Yang C, Ji F, Gao H, Yang M, Zhu T, Li J, Wang K. Visualization positioning-guided biopsy of suspicious breast microcalcifications: a retrospective cohort study. Ann Transl Med 2021;9(21):1620. doi: 10.21037/atm-21-4496 of Malignancy for the Incomplete Retrieval of Suspicious Calcifications Diagnosed as Flat Epithelial Atypia or Atypical Ductal Hyperplasia by Stereotactic VacuumAssisted Breast Biopsy. Korean J Radiol 2020;21:1220-9.

(English Language Editor: C. Betlzar) 